\title{
Advancing scientific knowledge in times of pandemics
}

\author{
Nicolas Vabret ${ }^{\bowtie}$, Robert Samstein, Nicolas Fernandez, Miriam Merad \\ and The Sinai Immunology Review Project*
}

Researchers at the Precision Immunology Institute at the Icahn School of Medicine (PrIISM), New York, describe their contribution to the global research effort against COVID-19 by trying to separate signal from noise in the preprint arena.

Since its reported emergence in Wuhan, China, in December 2019, the fast-growing pace of the COVID-19 pandemic has been challenging the capacities of healthcare systems worldwide. Our research institute, the Precision Immunology Institute at the Icahn School of Medicine (PrIISM) is located within the Mount Sinai Health System in New York, a city that is disproportionately affected by COVID-19. With non-COVID-19 research being progressively halted, several immunology laboratories here have started to address COVID-19related research efforts, such as studying the interactions of SARS-CoV-2 with the immune system, understanding the immunopathology that it causes, and developing new therapies and vaccine strategies against the virus.

In parallel to these research programmes, we initiated an institutional effort to provide critical reviews of COVID-19 articles posted daily on the preprint servers bioRxiv and medRxiv ${ }^{1}$, which we call the Sinai Immunology Review Project. This effort was designed to meet two needs. The first is a desire from our students, postdocs, fellows and faculty members to contribute to the global research effort and harness their knowledge and skills to help the scientific community. The second stems from a challenging consequence of the growing importance of preprints in scientific communication. The swift and open dissemination of information offered by preprints has already proved crucial in providing rapid scientific responses to the emerging public threat of viral outbreaks $s^{2,3}$. However, the ambition of preprint servers was never to replace the peer-review process. The spread of the COVID-19 pandemic has been matched by an impressive rise in related publications in both preprint servers and peer-reviewed journals. At a time when journal editors and invited reviewers are more solicited than ever, we decided that it is the responsibility of the scientific community to help curate this unprecedented flow of scientific data. Reviewing preprints benefits both the authors and the scientific community and provides the general public with access to scientific discussion, helping to reinforce scientific credibility ${ }^{4}$.

Our workflow ranks each COVID-19-related preprint (totalling 1,772 to date) according to its immunological relevance. The most relevant papers are then reviewed in detail by trainees and validated by a faculty member. Validated reviews (118 to date) are then posted alongside the corresponding article on the preprint server. A PrIISM scientist, Nicolas Fernandez, built a dedicated website to host and integrate all of our reviews (see Related links), which are further shared with other pre-publication reviewing initiatives ${ }^{4}$. We have also launched a collaboration with Nature Reviews Immunology to publish short summaries of preprints of particular interest in a weekly column.

Despite its flaws, peer-reviewing is an integral part of a scientist's mission to the academic community and to the public. Jean Monet, one of the founding fathers of the European Union, stated: "People only accept changes in necessity and see necessity only in crisis". The COVID-19 pandemic and its prolific scientific response provide a unique opportunity for our community to experiment with new methods of peer-reviewing scientific communications at scale, and the Sinai immunology community is stepping up to the challenge.

\footnotetext{
Sever, R. et al. Preprint at bioRxiv https://doi.org/10.1101/833400 (2019).

2. Johansson, M. A., Reich, N. G., Meyers, L. A. \& Lipsitch, M.

PLoS Med. 15, e1002549 (2018).

3. Majumder, M. S. \& Mandl, K. D. Lancet Glob. Health

https://doi.org/10.1016/S2214-109X(20)30113-3 (2020).

4. Hindle, S. \& Saderi, D. elife https://elifesciences.org/

labs/57d6b284/prereview-a-new-resource-for-the-collaborative-

review-of-preprints (2017)

5. Monnet, J. Mémoires (Fayard, 1988)
}

Competing interests

The authors declare no competing interests.

RELATED LINKS

Reviewing COVID-19 SARS-CoV-2 preprints from medRxiv and bioRxiv: https://bit.ly/covid-19-sinai-reviews 